\title{
Quench Performance of the LHC Insertion Magnets
}

\author{
Nuria Catalan Lasheras, Walter Venturini Delsolaro, Andrzej Siemko, Ranko Ostojic, and Glyn Kirby
}

\begin{abstract}
After final installation in the LHC tunnel, the MQM and MQY quadrupole magnets of the LHC insertions are now being commissioned to their nominal currents. These two types of magnets operate at $1.9 \mathrm{~K}$ and $4.5 \mathrm{~K}$ and with nominal currents ranging from 3600 A to $5390 \mathrm{~A}$. From the very first acceptance tests of the bare magnets coming from the manufacturers, they have been powered using different cycles, in different configurations, at different temperatures and in different tests facilities. In this paper we present the global results of these powering tests. We aim at separating common from individual features of these groups of magnets. Temperature dependence of the training, temperature margin, and ultimate current can be extracted from these tests. As these magnets are used to match the optics and the dispersion in the machine, the projected ultimate current at which they can be operated is critical in view of operation of LHC.
\end{abstract}

Index Terms-LHC, power tests, quench training, superconducting accelerator magnets.

\section{INTRODUCTION}

$\mathbf{T}$ HE LHC will be the most powerful particle accelerator in the world accelerating two proton beams at an energy of $7 \mathrm{TeV}$ and having them collide at the center of four gigantic detectors [1]. More than 1700 main dipole and quadrupole superconducting magnets ensure that the proton beams stay inside the vacuum enclosure of $27 \mathrm{~km}$ in circumference. The so called insertion magnets of the LHC machine are individually powered quadrupoles located in the long straight sections which help match the optics functions of the beam in the machine [2]. They are assembled using two types of twin aperture magnets, the $56 \mathrm{~mm}$ aperture MQM and the $70 \mathrm{~mm}$ aperture MQY. Both have separated collared coil, and a common yoke structure. The MQY-type magnets sit in stand-alone cryostats filled with boiling helium at $4.5 \mathrm{~K}$. The MQM-type magnets sit either in stand-alone cryostats also at $4.5 \mathrm{~K}$ or in the continuous arc cryostat at $1.9 \mathrm{~K}$ super-fluid helium. In Table I the main features of these magnet types referring to operating conditions are displayed, including the nominal and ultimate current.

These magnets were manufactured in European companies and shipped to CERN as yoked apertures. The majority of the yoked apertures were tested as part of the acceptance tests for quench performance in a vertical cryostat facility at CERN commonly named "Block 4" [3]. After assembling them in cold

Manuscript received August 17, 2008. Current version published July 15, 2009.

The authors are with the European Organization for Nuclear Research, CERN, Geneva 23, CH-1211 Switzerland (e-mail: nuria.catalan. lasheras@cern.ch; Walter.Venturini@cern.ch; Andrzej.Siemko@cern.ch; Ranko.Ostojic@cern.ch; Glyn.Kirby@cern.ch).

Color versions of one or more of the figures in this paper are available online at http://ieeexplore.iee.org.

Digital Object Identifier 10.1109/TASC.2009.2017708
TABLE I

OPERATING CONDITIONS FOR THE INSERTION MAGNETS IN THE LHC

\begin{tabular}{ccccc}
\hline \hline Magnet type & $\begin{array}{c}\text { Operating } \\
\text { temperature } \\
{[\mathbf{K}]}\end{array}$ & $\begin{array}{c}\text { Nominal } \\
\text { Current } \\
{[\mathbf{A}]}\end{array}$ & $\begin{array}{c}\text { Ultimate } \\
\text { current } \\
{[\mathbf{A}]}\end{array}$ & $\begin{array}{c}\text { \# of } \\
\text { magnets } \\
\text { in LHC }\end{array}$ \\
\hline MQM & 1.9 & 5390 & 5820 & 46 \\
MQM & 4.5 & 4310 & 4650 & 14 \\
MQY & 4.5 & 3610 & 3900 & 18 \\
\hline \hline
\end{tabular}

masses and inserting in final cryostats, the completed cryo-magnets were tested again for quench performance and, in some cases, magnetic measurements were performed in the SM18 magnet testing facility [4]. The cryo-magnets were then lowered in the tunnel, interconnected to each other and finally connected to the cryogenic system.

During the last year, the sector by sector commissioning of the LHC superconducting circuits has taken place and two thirds of the insertion quadrupoles have been powered to their nominal currents.

The results of these tests have been analysed to look at the predictability of the magnet training in the machine. Section II briefly describes the testing conditions in the test facilities as well as the operating conditions in the machine. Section III presents a comparison of the quench training performance in terms of the number of quenches per magnet experienced at the different stages. The special case of the quench training of magnets operating at $4.5 \mathrm{~K}$ is developed in Section IV.

\section{Testing AND Operating Conditions}

\section{A. Block 4 Testing Conditions}

In the CERN vertical cryostat test facility the yoked twin apertures magnets were tested in a helium bath at $4.3 \mathrm{~K}$ for the MQY-type or in a super-fluid helium bath at $1.9 \mathrm{~K}$ for the MQM-type. At the end of the production, some of the MQM magnets were tested at $4.3 \mathrm{~K}$ but not necessarily the ones operating in the LHC at that temperature. The longest MQM type magnet ( $4.8 \mathrm{~m}$ long) could not be tested in this test facility. As part of the tests, magnetic measurements were performed using rotating coil technique. Voltage taps were used for the quench detection and traceability of the origin of the quenches. The magnets were systematically powered to their ultimate currents defined as $8 \%$ above the nominal currents. This corresponds to an ultimate energy of the LHC proton beam of $7.54 \mathrm{TeV}$ and corresponds to $9 \mathrm{~T}$ in the arc dipole.

\section{B. SM18 Testing Conditions}

The fully assembled and cryostated magnets were connected to a cryogenic and electrical feed box and tested in one of the 


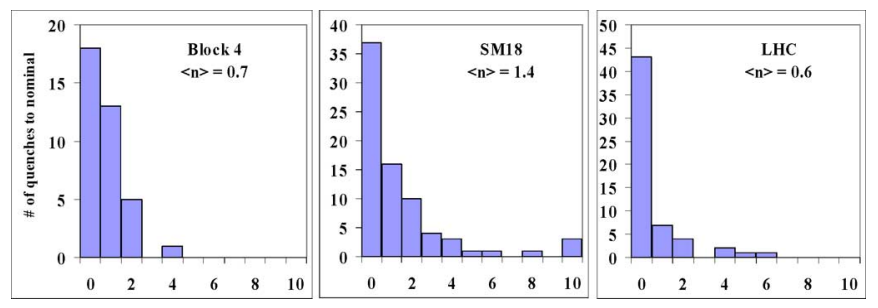

Fig. 1. Histogram and average value of the number of quenches to reach nominal current in Block 4 and SM18, during acceptance tests and in the LHC during commissioning.

dedicated horizontal test benches in the SM18 test facility. For magnets operating at $1.9 \mathrm{~K}$, the temperature was controlled by the helium vapor pressure in the cryogenic heat exchanger integrated in the magnet yoke and measured by a sensor installed in the helium enclosure. The temperature of the magnets operating at $4.5 \mathrm{~K}$ was controlled by the pressure of the main He I bath and measured by a temperature sensor installed outside the magnet helium enclosure in thermal contact with the bottom part of the level gauge. The He I bath temperature for those magnets without heat exchanger could only be lowered to $4.6 \mathrm{~K}$ (1.35 bar). Some magnets that operate in the LHC at $1.9 \mathrm{~K}$ but are not equipped with a heat exchanger were tested in SM18 only at 4.6 $\mathrm{K}$.

More than one yoked aperture may be included in a cryomagnet. Like in the Block 4 test facility the origin of the quench was traced back to proper aperture and yoked magnet by means of the voltage taps.

\section{LHC Operating Conditions}

The operating temperature for insertion magnets in the LHC is $1.9 \mathrm{~K}$ for the continuous cryostat in the arc and $4.5 \mathrm{~K}$ for the stand alone cryo-magnets. The temperature of the stand alone magnets is controlled by the pressure of the vapor exhaust (1.3 bar) and shows small variations. The slope of the tunnel in the LHC insertions varies from $0.36 \%$ to $1.39 \%$ depending on the location of the magnet. The protection system does not make use of all the voltage taps available. It is possible to discriminate in which aperture the quench originated but not in which magnet if there is more than one.

\section{The Quench TRAINING}

All the insertion magnets were accepted after the power performance tests. Nominal current was reached rather easily and ultimate current was reached for most of the magnets. Fig. 1 shows the distribution of the number of quenches to reach nominal current in the two test facilities and in the LHC tunnel. The number of training quenches for each assembled magnet is taken as the sum of the quenches experienced in each magnet and aperture if tested separately. The population of the samples is different as not all the magnets were tested in the vertical cryostat and not every magnet has been powered yet in the LHC machine.

These plots clearly show that the average number of quenches to reach the nominal current decreases between both testing facilities and the LHC. The spread is also reduced. This is a typical sign of "memory" after magnet quench training. However,

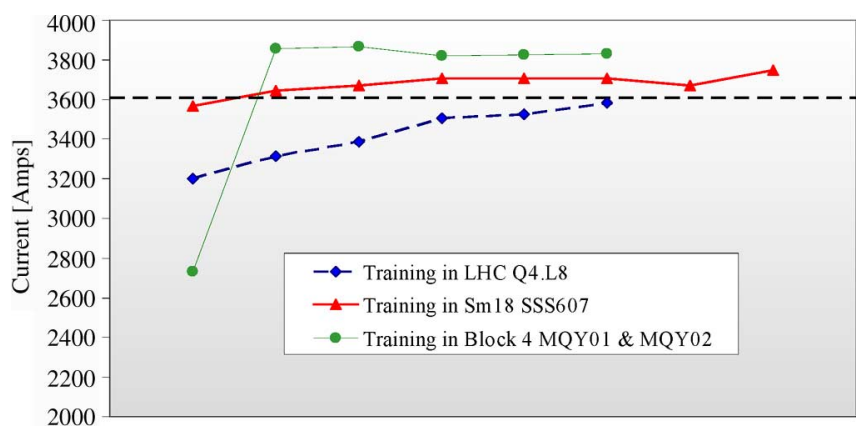

Fig. 2. Training of Q4.L8 in the LHC, SM18 and Block 4.

the quench training in Block 4 was observed to be much shorter than in SM18.

A closer look at the data by checking magnet by magnet shows that the power tests for some of them failed to predict a long training in the machine. Three magnets experienced more than three quenches during commissioning. Fig. 2 shows the training of magnet Q4.L8 and its corresponding components (whose series number is SSS607 made with magnets MQY01 and MQY02). The training seems to degrade at each stage. This magnet is the oldest of its kind and it was fabricated using the two first pre-series magnets. The Block 4 training curve shows no improvement after the second quench. The degradation of the quench performance is yet unexplained. It may be related to the stability of the mechanical assembly and transport of the magnet from the test facilities to the LHC tunnel.

For the other two magnets that had more than three quenches in the LHC, the training was not performed in Block 4 or was done at a different temperature. However, the number of quenches to nominal current during the acceptance tests in SM18 was lower than what was experienced in LHC for both cases.

\section{Quench Training to Ultimate CuRRent}

In the Block 4 and SM18 test facilities, magnets were trained up to ultimate current (about $8 \%$ above nominal, see Table I). The average number of quenches per magnet to reach ultimate current was equal to 3 in block 4 and 5.4 in SM18. In Block 4, the number of quenches to reach ultimate current at $1.9 \mathrm{~K}$ and $4.5 \mathrm{~K}$ is similar within $30 \%$. Training at $1.9 \mathrm{~K}$ is always faster thanks to the increased stability margin in super-fluid helium. However, the dependence of the number of training quenches on operational temperature observed in SM18 was much more dramatic. Fig. 3 shows the distribution of quenches for magnets tested at $1.9 \mathrm{~K}$ and $4.5 \mathrm{~K}$. The average number of quenches to ultimate at $1.9 \mathrm{~K}$ is 1.5 whereas at $4.5 \mathrm{~K}$ is 10.7 .

To be able to compare the quench training results for both the MQM and MQY magnets, the short sample current is used to normalize the quench currents. It is calculated from the critical surface of MQM and MQY magnet cables derived from strand measurements taken during cable production. The magnetic field for the calculation is given by the 2D model in ROXIE software [5] in any given conductor for each magnet type. We use the average short sample current from the strand measurements but some magnets may well have a lower short sample current coming from the cable characteristics. 


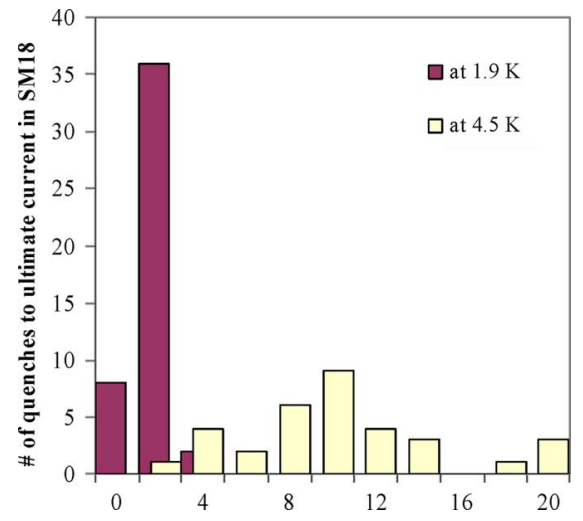

Fig. 3. Distribution of the number of quenches to nominal for magnets tested at $1.9 \mathrm{~K}$ and $4.5 \mathrm{~K}$ in SM18.
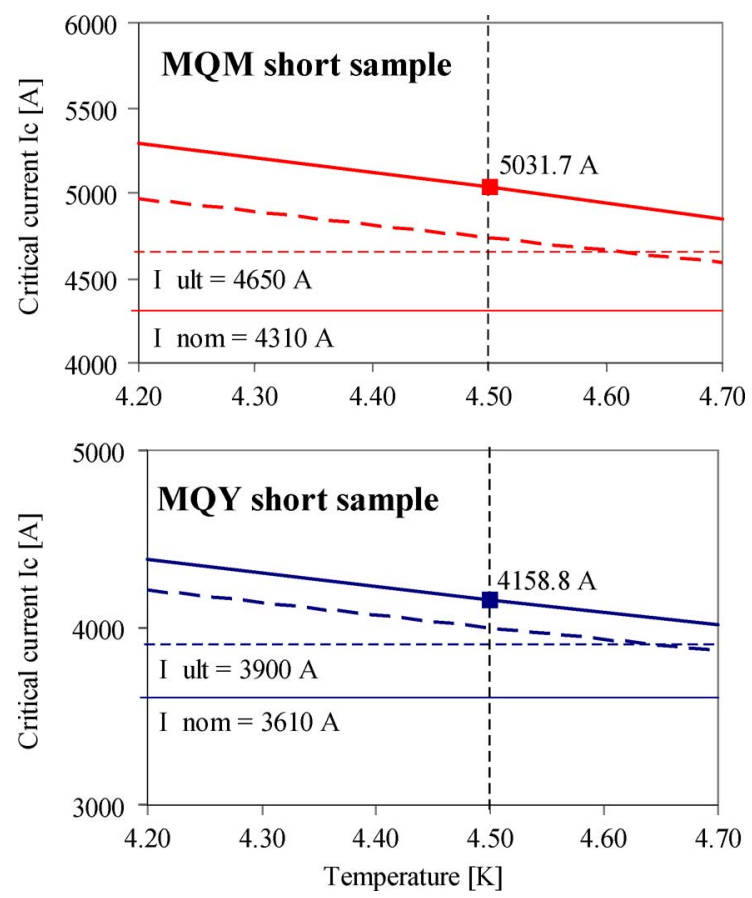

Fig. 4. Short sample calculation for MQM and MQY magnets at $4.5 \mathrm{~K}$ and at the peak field in the coil. The top line represents average measured values in strands. The lower limit represents the minimum measured short sample current.

The dependence of the critical current of the MQM and MQY short sample as a function of temperature is shown in Fig. 4. The temperature margin at $4.5 \mathrm{~K}$ is very small even for the average measured short-sample current. For some magnets this temperature margin is even lower. The load-line percentage and the temperature margin calculated by ROXIE are shown in Table II.

The quench training results for all the MQM and MQY magnets normalized to the short sample current is shown in Fig. 5. For MQM magnets, the spread of the number of training quenches to reach ultimate current is very large but we can distinguish two magnets for which the training was sensibly slower. There is no noticeable difference between the training of MQM or MQY magnets. Magnets that underwent a thermal
TABLE II

LoAd-Line Percentage and Temperature Margin Computed by RoXie FOR DIFFERENT TYPES OF LHC INSERTION MAGNETS

\begin{tabular}{cccc}
\hline \hline Magnet type & $\begin{array}{c}\text { Operating } \\
\text { temperature } \\
{[\mathbf{K}]}\end{array}$ & $\begin{array}{c}\text { Working } \\
\text { point in the } \\
\text { load-line [\%] }\end{array}$ & $\begin{array}{c}\text { Temperature } \\
\text { margin } \\
{[\mathbf{K}]}\end{array}$ \\
\hline MQM & 1.9 & 80 & 2.1 \\
MQM & 4.5 & 87 & 0.77 \\
MQY & 4.5 & 84 & 0.93 \\
\hline \hline
\end{tabular}
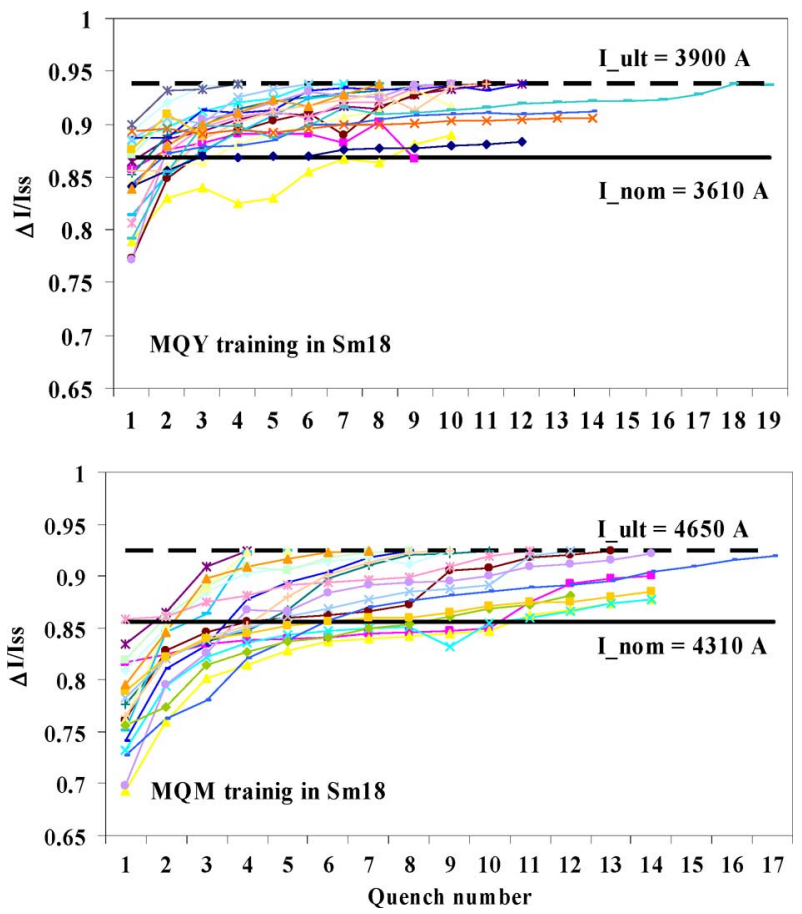

Fig. 5. Training curves to ultimate current normalized by the short sample current, for all the MQM (bottom) and MQY-type (top) magnets in SM18. The test temperature is $4.5 \mathrm{~K}$.

cycle are also included and in general did not show a faster training.

Another observation that singles out the training in SM18 is the training speed. The steps in current between quenches of a magnet follow a different distribution in the two test facilities and the LHC. Whereas consecutive quenches in Block 4 have an average current increase of $3.74 \%$ of the short sample current, the current increase in SM18 is only $1.44 \%$ of the short sample. The same current increase in the LHC is, in average, $3.0 \%$. The statistical error is indeed larger for the Block 4 and LHC due to the smaller statistic sample.

It is clear that the higher temperature in the SM18 test benches as compared to the Block 4 or the LHC plays a role in the longer training. The probability that similar small energy releases will raise the temperature over the critical surface is significantly higher at $4.6 \mathrm{~K}$ then at $4.5 \mathrm{~K}$. Another possible explanation for the difference in current steps is the fact that benches in SM18 are completely horizontal. Any gas bubble in the helium bath may be trapped next to the quadrupole bore. On the other hand, 


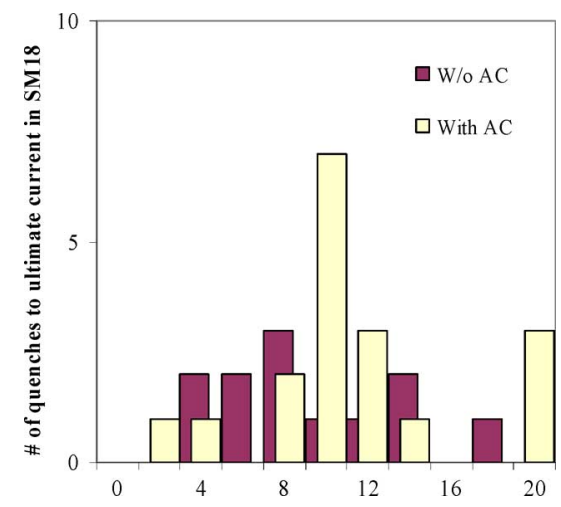

Fig. 6. Distribution of the number of quenches to ultimate current at $4.5 \mathrm{~K}$. The two distributions correspond to magnets measured with or without an anticryostat.

in Block 4, where the cryostat is vertical or in the LHC, where the tunnel has a certain slope, the helium gas circulation is ensured.

The presence of anti-cryostats to measure magnetic properties may affect the training behavior as it was observed during the training of separation dipoles in Brookhaven National Laboratory [6]. They account for an extra heat load as they are open to the atmospheric pressure. Indeed, the number of quenches to ultimate current was higher for magnets in which an anti-cryostat was mounted (see Fig. 6).

To verify this hypothesis, the measurement device was removed and the anti-cryostat was emptied to provide temperature insulation during the powering tests of a small number of magnets. The speed and length of the training at $4.5 \mathrm{~K}$ did not improve significantly, ruling out this hypothesis.

\section{CONCLUSION}

The training behavior of the LHC insertion quadrupole magnets was generally very good. It exceeded the requirements for the machine. All magnets coming from the manufacturers were accepted after power tests. After the training campaigns in the test facilities at CERN, $73 \%$ of the magnets reached nominal current without a quench and $13 \%$ experience a single quench. Only two magnets operating at $1.9 \mathrm{~K}$ experienced a single training quench in the LHC machine during hardware commissioning.

Longer training is observed for magnets operating at $4.5 \mathrm{~K}$ when powered to ultimate current, in particular in SM18. This is partially explained by the higher temperature $(4.6 \mathrm{~K}$ instead of $4.5 \mathrm{~K}$ ).

\section{REFERENCES}

[1] O. Brüning, Ed. et al., LHC Design Report CERN. Geneva, 2004 [Online]. Available: http://ab-div.web.cern.ch/ab-div/Publications/LHC-DesignReport.html

[2] H. Prin et al., "Production of the superconducting matching quadrupoles for the LHC insertions," in 19th International Conference on Magnet Technology, Genova, Italy, Sep. 18-23, 2005.

[3] W. Venturini Delsolaro et al., "The test facility for the short prototypes of the LHC superconducting magnets," in AIP Conf. Proc., 2002, vol. 613, p. 106.

[4] A. Siemko and P. Pugnat, "Performance evaluation and quality assurance management during the series power tests of LHC main lattice magnets," IEEE Trans. Appl. Sup, vol. 18, no. 2, pp. 126-131, June 2008.

[5] S. Russenschuck, CERN, ROXIE: A Computer Code for the Integrated Design of Accelerator Magnets LHC-Project-Report-276, 1999.

[6] J. F. Muratore et al., "Test results for LHC insertion region dipole magnets," in 21st IEEE Particle Accelerator Conference, Knoxville, TN, USA, May 16-20, 2005, p. 3106. 